DOI: https://doi.org/10.11144/Javeriana.umed58-1.vsaf

\title{
Valores séricos de ácido fólico en un grupo de pacientes mayores de 18 años de edad en un hospital de alta complejidad *
}

\section{Serum Folic Acid Values in a Group of Patients over 18 Years of Age in a Highly Complex Hospital}

\author{
Lyda Salamanca ${ }^{1}$ \\ Pontificia Universidad Javeriana, Bogotá, Colombia \\ Adriana ORdÓÑEZ ${ }^{2}$ \\ Pontificia Universidad Javeriana, Colombia \\ Claudia Cardozo ${ }^{3}$ \\ Hospital Universitario San Ignacio, Colombia \\ Fernando SuÁrez-OBando 4 \\ Pontificia Universidad Javeriana, Colombia
}

\footnotetext{
1 Bacterióloga, Pontificia Universidad Javeriana, Bogotá, Colombia.

2 Profesor(a), Instituto de Genética Humana, Facultad de Medicina, Pontificia Universidad Javeriana, Bogotá, Colombia.

3 Miembro del Laboratorio Clínico, Hospital Universitario San Ignacio, Bogotá, Colombia.

4 Profesor, Instituto de Genética Humana, Facultad de Medicina, Pontificia Universidad Javeriana, Bogotá, Colombia.
}

Cómo citar: Salamanca L, Ordóñez A, Cardozo C, Suárez-Obando F. Valores séricos de ácido fólico en un grupo de pacientes mayores de 18 años de edad en un hospital de alta complejidad. Univ Med. 2017;58(1): 1-7. https://doi.org/10.11144/ Javeriana.umed58-1.vsaf

\section{RESUMEN}

Objetivo: establecer las concentraciones séricas de ácido fólico en un grupo de pacientes adultos. Métodos: medición del ácido fólico con inmunoanálisis en 1797 sujetos. Se calcularon medidas de tendencia central e índice de confianza al $95 \%$. Se compararon grupos etarios y sexos con pruebas no paramétricas. Resultados: la media global de ácido fólico sérico fue: 11,61 ng/ml (DE: 6,05). La media en mujeres fue de $11,87 \mathrm{ng} / \mathrm{ml}$ (DE: 6,09). La media en hombres fue de 11,31 ng/ml (DE: 6). Se reportaron los percentiles 5, 50 y 95 por sexo y edad. En el $2 \%$ de los casos se encontró ácido fólico $<2 \mathrm{ng} / \mathrm{ml}$ (2\% de hombres y $3 \%$ de mujeres). Discusión: las concentraciones séricas de ácido fólico son mayores en mujeres que en hombres $(p=0,024)$, lo que contrasta con la noción de déficit del micronutriente en el sexo femenino. Conclusiones: las cantidades descritas son un comparador adecuado para mediciones en muestras de población general.

Palabras clave

ácido fólico; valores de referencia; vitaminas.

\begin{abstract}
Objective: To establish the serum levels of folic acid in a group of adult patients. Methods: Measurement of folic acid using immunoassay in 1797 subjects. Measures of central tendency and 95\% CI were calculated. Age groups and genders were compared with non-parametric tests. Results: The overall mean serum folate was: $11.61 \mathrm{ng} / \mathrm{ml}$ (SD: 6.05). The average for women was: $11.87 \mathrm{ng} / \mathrm{ml}$ (SD: 6.09). The average for men was: 11.31 $\mathrm{ng} / \mathrm{ml}$ (SD: 6). Percentiles 5, 50, and 95 by sex and age are reported. In $2 \%$ of cases folic acid was found $<2 \mathrm{ng} / \mathrm{ml}$ ( $2 \%$ of men and $3 \%$ women). Discussion: Serum levels of folic acid are higher in women than in men $(p=0.024)$, which contrasts with the notion of micronutrient deficiency in females. Conclusions: The presented measurements are an appropriate comparator described for measurements in general population samples.
\end{abstract}


Keywords

folic acid; reference values; vitamins.

\section{Introducción}

El ácido fólico o ácido pteroil-L-glutámico es una vitamina hidrosoluble del complejo de la vitamina B (vitamina B9), necesaria para diversos procesos bioquímicos, como la formación de proteínas estructurales, síntesis de nucleótidos y regulación epigenética [1]. Se encuentra en alimentos como las verduras de hoja verde, legumbres, frutos secos y granos enteros como las almendras [2]. El ácido fólico es de particular interés clínico por su relación con la anemia megaloblástica y diversos trastornos neurológicos relacionados con la carencia de la vitamina [3,4], suplencia perinatal y prevención de defectos del tubo neural [5], metabolismo de la homocisteína y su asociación con eventos vasculares cardiacos y cerebrales [6] y su relación con la disminución del riesgo a padecer diversos tipos de cáncer [7].

En Colombia hay un consumo deficitario de ácido fólico en mujeres en edad fértil $[8,9]$ y entre mujeres universitarias existe una escasa percepción sobre su importancia en la salud materna fetal [10]. Se estima un déficit nutricional de ácido fólico junto con otras vitaminas del complejo B [11]; sin embargo, no se han medido sus concentraciones en muestras poblacionales o en grupos de mujeres no embarazadas. Así mismo, aunque se ha explorado la relación entre el ácido fólico, el metabolismo de la homocisteína, la vitamina B12 y el riesgo cardiovascular en diversas poblaciones [12,13], no se han determinado las concentraciones de este ácido en muestras poblacionales masculinas del país.

Dada la posibilidad del déficit de este micronutriente y teniendo acceso a datos de laboratorio clínico de una población cautiva, el objetivo de este estudio fue establecer las concentraciones séricas de ácido fólico en un grupo de pacientes que asistió al laboratorio de un hospital de alta complejidad. Establecer esto permite obtener una línea de base para contrastar los valores séricos de ácido fólico con otras poblaciones de interés.

\section{Materiales y métodos}

Se obtuvo una base de datos con un total de 2270 sujetos a quienes se les midieron las concentraciones de ácido fólico, entre enero del 2009 y diciembre del 2011, en el Laboratorio Clínico del Hospital Universitario San Ignacio (HUSI), mediante la técnica de inmunoanálisis quimioluminiscente con micropartículas (ChemiFlex), integrado en una plataforma Architect de Abbott Diagnostics. Las variables estudiadas fueron: sexo (masculino y femenino), edad (años cumplidos), concentraciones de ácido fólico sérico en la sangre periférica $(\mathrm{ng} / \mathrm{ml})$, hemoglobina $(\mathrm{g} / \mathrm{dl})$ y hematocrito (\%). Se calcularon medidas de tendencia central, intervalo de confianza al $95 \%$ (IC-95\%) y percentiles 5, 50 y 95. Se empleó el test de normalidad de Shapiro-Wilk para evaluar la distribución de valores, luego de lo cual se determinó el uso de la prueba de la mediana para comparar los grupos. Se consideraron valores normales a los valores de referencia reportados por el laboratorio: ácido fólico: 3 a $17 \mathrm{ng} / \mathrm{ml}$; hemoglobina en mujeres: 11,5-14,5 $\mathrm{g} / \mathrm{dl}$; hemoglobina en hombres: 13,5-16,0 g/dl; hematocrito en mujeres: $37-47 \%$ y en hombres: 40-54\%. Se presentan los datos en tablas, gráficos de cajas y curvas de percentiles.

\section{Resultados}

Se eliminaron 303 registros, por no tener valores de hemoglobina y hematocrito; 116, por no tener registrado el sexo, y 54 sujetos menores de edad, para una muestra final de 1797 sujetos (masculino: 863 sujetos; femenino: 934 sujetos). Solo se analizaron las solicitudes de análisis que incluyeran hemoglobina, hematocrito y ácido fólico. Se identificaron los sujetos por la nota de remisión y no se tuvo acceso a la historia clínica. Así, se desconocía la razón específica para la solicitud de los exámenes. Solo se sabía el servicio que lo enviaba, y en 
el caso de la muestra de población femenina, eran mujeres no embarazadas. Los pacientes estudiados fueron enviados al laboratorio desde servicios ambulatorios y consulta externa en 1685 casos $(93,7 \%)$ y desde hospitalización en 113 casos $(6,3 \%)$.

El test de normalidad de Shapiro-Wilk indicó una distribución de los valores de ácido fólico diferente a la normal $(\mathrm{W}=0,88241 ; \mathrm{p}=0,0)$. En la figura 1 se aprecia la distribución por sexo y rangos de edad, según medias. El valor promedio de ácido fólico sérico en la muestra estudiada fue de 11,61 ng/ml (DE: 6,05; mínimo: 0,3 ng/ ml; máximo: $42 \mathrm{ng} / \mathrm{ml}$; mediana: $11,1 \mathrm{ng} / \mathrm{ml})$. El promedio de ácido fólico sérico en mujeres fue de 11,87 ng/ml (DE: 6,09; mediana: 11,5). El promedio de ácido fólico sérico en hombres fue de 11,31 ng/ml (DE: 6; mediana: 10,6).

Figura 1.Concentraciones de ácido fólico sérico por sexo y rango de edad (HUSI 2007-2011)

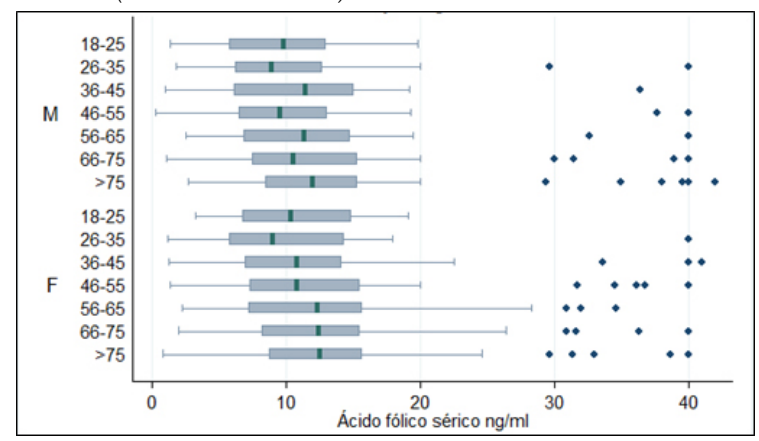

Nota: se aprecian valores por encima de $30 \mathrm{ng} /$ $\mathrm{ml}$ en casi todos los rangos de edad. Estos se encuentran usualmente en pacientes que reciben suplencias de la vitamina o en ciertas condiciones crónicas que pueden elevar las concentraciones séricas de la vitamina. M: masculino; F: femenino

En 43 casos (2\%) se encontró un valor de ácido fólico menor a $2 \mathrm{ng} / \mathrm{ml}$. La carencia se identificó en el $2 \%$ de los hombres ( 25 casos) y en el $3 \%$ de las mujeres (18 casos). En 17 hombres y 13 mujeres con bajas cantidades de ácido fólico se identificaron baja hemoglobina y hematocrito. Es decir, solo en 12 casos $(0,6 \%)$ se identificó la carencia de ácido fólico no asociada a anemia. Por otra parte, se encontraron 578 hombres (32\%) y 502 mujeres (28\%) con anemia y concentraciones séricas de ácido fólico normales. En 232 casos (12,8\%) se presentaron valores por encima de $17 \mathrm{ng} / \mathrm{ml}$ (100 hombres y 132 mujeres). La distribución de valores anormales se presenta en la tabla 1.

Tabla 1. Sujetos con concentraciones séricas anormales de ácido fólico según sexo y rango de edad

\begin{tabular}{|l|l|l|l|l|l|l|l|l|}
\hline \multicolumn{7}{|c|}{ Concentraciones séricas de ácido fólico < 3 ng/ml (n = 43) } \\
\hline Edad & $18-25$ & $26-35$ & $36-45$ & $46-55$ & $56-65$ & $66-75$ & $\begin{array}{l}75 \\
75\end{array}$ \\
\hline $\begin{array}{l}\text { Hombres } \\
\text { (n [\%]) }\end{array}$ & $1(2,3)$ & $7(16)$ & $2(4,6)$ & $\begin{array}{l}6 \\
(13,9)\end{array}$ & $4(9,3)$ & $4(9,3)$ & $\begin{array}{l}1 \\
(2,3)\end{array}$ \\
\hline $\begin{array}{l}\text { Mujeres } \\
\text { (n [\%]) }\end{array}$ & $0(0)$ & $1(2,3)$ & $3(6,8)$ & $\begin{array}{l}5 \\
(11,6)\end{array}$ & $4(9,3)$ & $2(4,6)$ & $\begin{array}{l}3 \\
(6,8)\end{array}$ \\
\hline Concentraciones séricas de ácido fólico > $\mathbf{1 7} \mathbf{n g} / \mathbf{m l} \mathbf{( n = 2 3 2 )}$ \\
\hline Edad & $18-25$ & $26-35$ & $36-45$ & $46-55$ & $56-65$ & $66-75$ & $\begin{array}{l}> \\
75\end{array}$ \\
\hline $\begin{array}{l}\text { Hombres } \\
\text { (n [\%]) }\end{array}$ & $2(0,8)$ & $8(3,4)$ & $\begin{array}{l}10 \\
(4,3)\end{array}$ & $\begin{array}{l}11 \\
(4,7)\end{array}$ & $\begin{array}{l}18 \\
(7,7)\end{array}$ & $\begin{array}{l}23 \\
(9,9)\end{array}$ & $\begin{array}{l}28 \\
(12)\end{array}$ \\
\hline $\begin{array}{l}\text { Mujeres } \\
\text { (n [\%]) }\end{array}$ & $4(1,7)$ & $8(3,4)$ & $\begin{array}{l}10 \\
(4,3)\end{array}$ & $\begin{array}{l}25 \\
(10,7)\end{array}$ & $\begin{array}{l}28 \\
(12)\end{array}$ & $21(9)$ & $\begin{array}{l}35 \\
(15)\end{array}$ \\
\hline
\end{tabular}

La distribución de valores de ácido fólico por rango de edad se aprecia en la tabla 2. En las tablas 3 y 4 se aprecian los valores distribuidos por sexo. Los percentiles $\left(\mathrm{P}_{5}, \mathrm{P}_{50}\right.$ y $\left.\mathrm{P}_{95}\right)$ para la muestra total y para cada sexo se aprecian en las figuras 2,3 y 4 .

Tabla 2. Concentraciones séricas de ácido fólico (muestra total)

\begin{tabular}{|l|l|l|l|l|l|}
\hline $\begin{array}{l}\text { Rangos } \\
\text { (años) }\end{array}$ & $\begin{array}{l}\text { Sujetos } \\
\text { (n [\%]) }\end{array}$ & Media & DE & $\begin{array}{l}\text { IC-95 } \\
\text { \% }\end{array}$ & Mediana \\
\hline $18-25$ & $\begin{array}{l}66 \\
(3,67)\end{array}$ & 10,33 & 4,71 & $\begin{array}{l}9,2- \\
11,5\end{array}$ & 10,3 \\
\hline $26-35$ & $\begin{array}{l}141 \\
(7,85)\end{array}$ & 10,22 & 6,13 & $\begin{array}{l}9,2- \\
11,2\end{array}$ & 9,0 \\
\hline $36-45$ & $\begin{array}{l}181 \\
(10,07)\end{array}$ & 11,23 & 6,15 & $\begin{array}{l}10,3- \\
12,1\end{array}$ & 10,9 \\
\hline $46-55$ & $\begin{array}{l}308 \\
(17,14)\end{array}$ & 11,01 & 6,05 & $\begin{array}{l}10,3- \\
11,7\end{array}$ & 10,1 \\
\hline $56-65$ & $\begin{array}{l}303 \\
(16,86)\end{array}$ & 11,59 & 5,71 & $\begin{array}{l}10,9- \\
12,2\end{array}$ & 11,6 \\
\hline $66-75$ & $\begin{array}{l}351 \\
(19,53)\end{array}$ & 11,94 & 6,34 & $\begin{array}{l}11,2- \\
12,6\end{array}$ & 11,4 \\
\hline 8 gt; 75 & $\begin{array}{l}447 \\
(24,87)\end{array}$ & 12,54 & 6,03 & $\begin{array}{l}11,98 \\
-13,1\end{array}$ & 12,3 \\
\hline
\end{tabular}


Lyda Salamanca, Adriana Ordóñez, Claudia Cardozo et al.

Tabla 3. Concentraciones séricas de ácido fólico (mujeres)

\begin{tabular}{|l|l|l|l|l|l|}
\hline $\begin{array}{c}\text { Rangos } \\
\text { (años) }\end{array}$ & $\begin{array}{l}\text { Sujetos } \\
\text { (n [\%]) }\end{array}$ & Media & DE & $\begin{array}{l}\text { IC-95 } \\
\text { \% }\end{array}$ & Mediana \\
\hline $18-25$ & $42(63)$ & 10,7 & 4,60 & $\begin{array}{l}9,3- \\
12,09\end{array}$ & 10,35 \\
\hline $26-35$ & $85(60)$ & 10,22 & 5,73 & $\begin{array}{l}8,9- \\
11,40\end{array}$ & 9,00 \\
\hline $36-45$ & $\begin{array}{l}100 \\
(55)\end{array}$ & 11,35 & 6,54 & $\begin{array}{l}10- \\
12,60\end{array}$ & 10,85 \\
\hline $46-55$ & $\begin{array}{l}163 \\
(52)\end{array}$ & 11,70 & 6,38 & $\begin{array}{l}10,7- \\
12,70\end{array}$ & 10,80 \\
\hline $56-65$ & $\begin{array}{l}154 \\
(50)\end{array}$ & 12,04 & 5,90 & $\begin{array}{l}11,1- \\
12,90\end{array}$ & 12,35 \\
\hline $66-75$ & $\begin{array}{l}146 \\
(41)\end{array}$ & 12,24 & 6,07 & $\begin{array}{l}11,2- \\
13,20\end{array}$ & 12,45 \\
\hline \&gt; 75 & $\begin{array}{l}244 \\
(54)\end{array}$ & 12,62 & 6,10 & $\begin{array}{l}11,8- \\
13,30\end{array}$ & 12,50 \\
\hline
\end{tabular}

Tabla 4. Concentraciones séricas de ácido fólico (hombres)

\begin{tabular}{|c|c|c|c|c|c|}
\hline $\begin{array}{c}\text { Rangos } \\
\text { (años) }\end{array}$ & $\begin{array}{c}\text { Sujetos } \\
\text { (n [\%]) }\end{array}$ & Media & $\mathrm{DE}$ & $\begin{array}{c}\text { IC-95 } \\
\%\end{array}$ & Mediana \\
\hline $18-25$ & $24(37)$ & 9,60 & 4,93 & $\begin{array}{l}7,6- \\
11,80\end{array}$ & 9,85 \\
\hline $26-35$ & $56(40)$ & 10,26 & 6,7 & $\begin{array}{l}8,4- \\
12,07\end{array}$ & 8,9 \\
\hline $36-45$ & $81(45)$ & 11,08 & 5,6 & $\begin{array}{l}9,8- \\
12,30\end{array}$ & 11,5 \\
\hline $46-55$ & $\begin{array}{l}145 \\
(48)\end{array}$ & 10,16 & 5,5 & $\begin{array}{l}9,2- \\
11,08\end{array}$ & 9,6 \\
\hline $56-65$ & $\begin{array}{l}149 \\
(50)\end{array}$ & 11,10 & 5,4 & $\begin{array}{l}10,2- \\
12\end{array}$ & 11,4 \\
\hline $66-75$ & $\begin{array}{l}205 \\
(59)\end{array}$ & 11,70 & 6,5 & $\begin{array}{l}10,8- \\
12,63\end{array}$ & 10,6 \\
\hline \&gt; 75 & $\begin{array}{l}203 \\
(45)\end{array}$ & 12,44 & 5,9 & $\begin{array}{l}11,6- \\
13,20\end{array}$ & 12,3 \\
\hline
\end{tabular}

Figura 2. Percentiles de las concentraciones séricas de ácido fólico por rango de edad (población total)

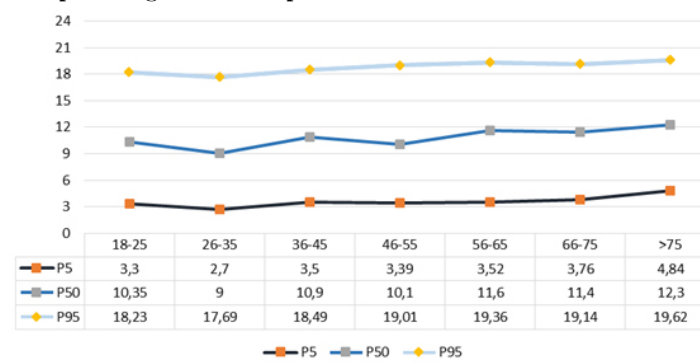

Figura 3. Percentiles de las concentraciones séricas de ácido fólico por rango de edad (población femenina)

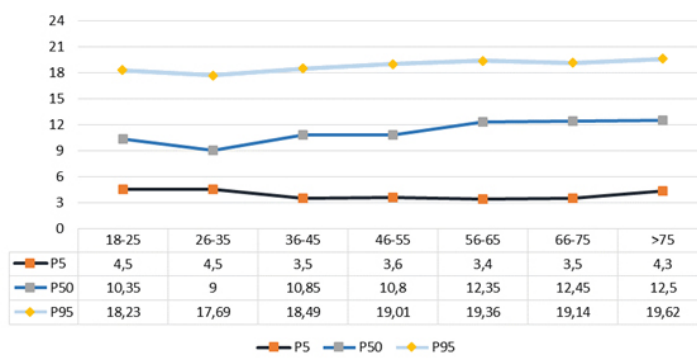

Figura 4. Percentiles de las concentraciones séricas de ácido fólico por rango de edad (población masculina)

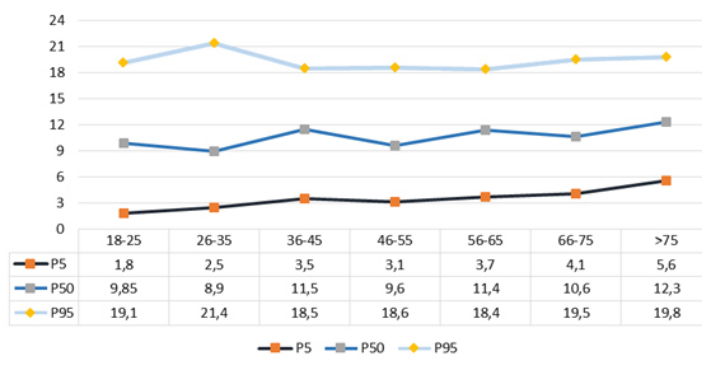

Al comparar las medianas de ácido fólico entre sexos, se encuentra una diferencia significativa (Pearson $\left.\chi^{2}=5,0764 ; \mathrm{p}=0,024\right)$, donde la mediana de la concentración de ácido fólico es mayor en mujeres. Al comparar tales concentraciones entre los rangos de edad en mujeres y hombres, se encuentra una diferencia significativa descrita en la tabla 5 .

Tabla 5. Comparación de medianas según rangos de edad en mujeres

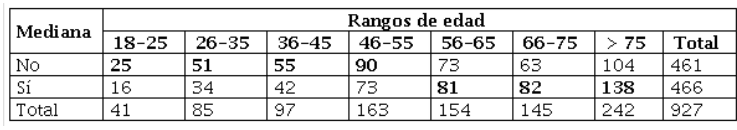

* Nota: las concentraciones séricas de ácido fólico por encima de la mediana se encuentran en población mayor a 55 años. Pearson $\chi^{2}=$ 16,$5465 ; \mathrm{p}=0,011$. Los valores exactos a la mediana se eliminaron del análisis.

La edad de las mujeres se transformó en una variable dicotómica que representó a las mujeres de edad fértil frente a las mujeres menopaúsicas $(18>$ edad mujeres $>55$ años). Al comparar las concentraciones séricas de ácido fólico entre ambos grupos, se encontró una diferencia significativa, teniendo las mujeres mayores de 18 años de edad $(n=390)$ una media 
de ácido fólico de 11,2 ng/ml (DE: 6,1; mediana: $10,4)$ y las mujeres mayores a 55 años $(n=544)$ una media de 12,35 ng/ml (DE: 6,03; mediana: 12,4; Pearson $\left.\chi^{2}=15,4104 ; \mathrm{p}=0,0\right)$.

De la misma forma, la edad de los hombres se transformó en una variable dicotómica que representó a los hombres con mayor riesgo cardiovascular según la edad (45 años $>$ edad hombre $>45$ años). Al comparar ambos grupos, no se encontró una diferencia significativa (tabla 6): los hombres menores a 45 años $(\mathrm{n}=161)$ alcanzaron una media sérica de ácido fólico de 11,43 ng/ml (DE: 6; mediana: 10,6) y los hombres mayores a 45 años $(\mathrm{n}=702)$ una media de 10,5 ng/ml (DE: 5,95; mediana: 10; Pearson $\left.\chi^{2}=0,4309 ; \mathrm{p}=0,5\right)$. Al comparar las cantidades de ácido fólico para el grupo total entre mayores y menores de 55 años, se encontró que los mayores de 55 (hombres y mujeres) tienen concentraciones significativamente mayores de ácido fólico que los menores de 55 (Pearson $\chi^{2}=$ 20,3126; $\mathrm{p}=0,0$ ).

Tabla 6. Comparación de medianas según rangos de edad en hombres

\begin{tabular}{|l|l|l|l|l|l|l|l|l|}
\hline \multirow{2}{*}{ Mediana } & \multicolumn{7}{|c|}{ Rangos de edad } \\
\cline { 2 - 9 } & $\mathbf{1 8 - 2 5}$ & $\mathbf{2 6 - 3 5}$ & $\mathbf{3 6 - 4 5}$ & $\mathbf{4 6 - 5 5}$ & $\mathbf{5 6 - 6 5}$ & $\mathbf{6 6 - 7 5}$ & $>75$ & Total \\
\hline No & $\mathbf{1 3}$ & 34 & 37 & $\mathbf{8 6}$ & 70 & 102 & 85 & 427 \\
\hline Sí & 11 & 21 & 44 & 50 & $\mathbf{7 9}$ & $\mathbf{1 0 1}$ & $\mathbf{1 1 6}$ & 431 \\
\hline Total & 24 & 55 & 81 & 145 & 149 & 203 & 201 & 858 \\
\hline
\end{tabular}

* Nota: las concentraciones séricas de ácido fólico por encima de la mediana se encuentran en población mayor a 55 años de edad. Pearson $\chi^{2}=14,1832 ; \mathrm{p}=0,028$. Los valores exactos a la mediana se eliminaron del análisis.

\section{Discusión}

La carencia del ácido fólico en las mujeres se asocia con un incremento de defectos del tubo neural durante el desarrollo gestacional de sus hijos [14]; así mismo, las concentraciones séricas disminuidas de la vitamina se relacionan con diversas anomalías del metabolismo de la homocisteína y un incremento con el riesgo incrementado de padecer enfermedad vascular y de desarrollar algunos tipos de cáncer $[15,16]$. En muestras de población pediátrica colombiana se han relacionado las concentraciones normales o aumentadas de vitaminas del complejo B con mejor desarrollo pondoestatural [17,18]. Por otra parte, se ha descrito que en pacientes colombianos con enfermedad cardiovascular se presentan valores significativamente menores de ácido fólico en suero frente a sus controles sanos [19]; también se han identificado diferencias de las cantidades de folato entre poblaciones. Por ejemplo, el estudio de Caicedo y colaboradores [20] estableció una prevalencia del $26 \%$ de deficiencia de folato eritrocitario en pacientes con malaria, lo que contrasta con una prevalencia de menos del $1 \%$ de la misma deficiencia, en población escolar pediátrica de Bogotá [21]. Sin embargo, no hay un referente en la literatura que describa localmente las concentraciones de ácido fólico en sujetos con diversos rangos de edad y que establezcan si hay diferencias según grupos etarios y sexo.

La prevalencia de déficit de ácido fólico sérico en el presente estudio fue del $2 \%$. La mayoría de los casos de deficiencia se presentó con anemia. Esto contrasta con deficiencias hasta del $43 \%$ en poblaciones de la Amazonia [22] o ausencias anormales en jóvenes africanos [23]. Llama la atención que las concentraciones séricas de ácido fólico son mayores en mujeres que en hombres, lo que contrasta con la noción de déficit del micronutriente en el sexo femenino, aspecto que se desprende de estudios en los cuales se señala que en las mujeres embarazadas las recomendaciones de ingesta de vitaminas son inapropiadas [24]. De acuerdo con la Encuesta Nacional de Demografía y Salud del 2010, el consumo de ácido fólico es mayor en mujeres con menor número de hijos, mayor nivel educativo y mejores condiciones socioeconómicas [8].

En el grupo estudiado, las concentraciones más altas de ácido fólico se presentan en las mujeres mayores de 55 años (tabla 5). Así mismo, las más elevadas en hombres se detectan en mayores de 55 años, lo que podría explicarse por algunas condiciones crónicas (como falla renal), por ser personas que reciben suplemento vitamínico o por alguna relación entre las cantidades detectadas y la fortificación de ciertos alimentos de consumo masivo en el país [11], tal como sucede en algunos Estados, donde el 
ácido fólico está elevado de forma secundaria a la fortificación de alimentos [25].

La presente investigación tuvo varias limitaciones: las características de salud de los sujetos se desconocieron, no hubo historia nutricional, no se contaba con los índices eritrocitarios de ácido fólico y se trataba de un grupo de personas que asistió a servicios de salud, donde los médicos tratantes consideraron la medición sérica de ácido fólico dentro una batería diagnóstica, de tal modo que el grupo estudiado representa personas cuyas muestras de sangre se analizaron, por razones médicas, en un laboratorio en un hospital de alta complejidad. Lo anterior hizo suponer que eran pacientes en algún momento de la atención clínica, sea diagnóstico o de tratamiento, en los cuales se encontró una baja prevalencia de déficit sérico de ácido fólico. Sin embargo, era una población cautiva con mediciones confiables hechas por un laboratorio de última tecnología, de tal modo que lo aquí descrito es un comparador adecuado para mediciones en muestras de población general, dado que se presentan las concentraciones por sexo y rangos de edad con sus respectivos percentiles.

\section{Referencias}

1. Anderson OS, Sant KE, Dolinoy DC Nutrition and epigenetics: An interplay of dietary methyl donors, one-carbon metabolism and DNA methylation. J Nutr Biochem. 2012;23(8):853-9.

2. McNulty H, Pentieva K. Folate bioavailability. Proc Nutr Soc. 2004;63(4):529-36.

3. Chandra J. Megaloblastic anemia: Back in focus. Indian J Pediatr. 2010;77(7):795-9.

4. Koike H, Takahashi M, Ohyama K, et al. Clinicopathologic features of folate-deficiency neuropathy. Neurology. 2015;84(10):1026-33.

5. Wilson RD; Genetics Committee, Wilson RD, Audibert F, Brock JA, et al. Preconception folic acid and multivitamin supplementation for the primary and secondary prevention of neural tube defects and other folic acid-sensitive congenital anomalies. J Obstet Gynaecol Can. 2015;37(6):534-52.

6. Reilly R, McNulty H, Pentieva K, Strain JJ, Ward M. MTHFR 677TT genotype and disease risk: is there a modulating role for B-vitamins? Proc Nutr Soc. 2014;73(1):47-56.

7. Kandi V, Vadakedath S. Effect of DNA methylation in various diseases and the probable protective role of nutrition: A mini-Review. Cureus. 2015;7 (8):e309.

8. Profamilia. Encuesta Nacional de Demografía y Salud. Bogotá; 2010.

9. Prada G, Soto A, Herrán O. Consumo de leguminosas en el departamento de Santander, Colombia: 2000-2003. Arch Latinoam Nutr. 2005;55(1):64-70.

10. Ordóñez A, Suárez-Obando F. Exploración sobre los conocimientos del ácido fólico y sus beneficios en la salud reproductiva en una población universitaria colombiana. Rev Colomb Obstet Ginecol. 2006;57(4):271-8.

11. Brito A, Mujica-Coopman MF, López de Romaña D, Cori H, Allen LH. Folate and vitamin B12 status in Latin America and the Caribbean: An update. Food Nutr Bull. 2015;36(2 Suppl):S109-18.

12. Bermúdez M, Briceño I, Gil F, Bernal J. Homocisteína y polimorfismos de cistationina $\beta$ sintasa y metilentetrahidrofolato reductasa en población sana de Colombia. Colomb Méd (Cali). 2006;37 (1):46-52.

13. Villarreal E, Forero Y, Poveda E, Baracaldo C, López E. Marcadores de riesgo cardiovascular en escolares de cinco departamentos de la región oriental en Colombia. Biomédica. 2008;28(1):38-49. doi: http:// dx.doi.org/10.7705/biomedica.v28i1.107

14. Vásquez AO, Suárez-Obando F. [Neural tube defects and folic acid: a historical overview of a highly successful preventive intervention]. Hist Cienc Saude Manguinhos. 2015;22(4):1157-72. doi: 10.1590/S0104-59702015005000002 
15. Waly MI, Ali A, Al-Nassri A, AlMukhaini M, Valliatte J, Al-Farsi Y. Low nourishment of B-vitamins is associated with hyperhomocysteinemia and oxidative stress in newly diagnosed cardiac patients. Exp Biol Med (Maywood). 2016 Jan;241(1):46-51. doi: $10.1177 / 1535370215596860$

16. Liew SC, Gupta ED. Methylenetetrahydrofolate reductase (MTHFR) C677T polymorphism: Epidemiology, metabolism and the associated diseases. Eur J Med Genet. 2015;58(1):1-10.

17. Duong MC, Mora-Plazas M, Marín C, Villamor E. Vitamin B-12 deficiency in children is associated with grade repetition and school absenteeism, independent of folate, iron, zinc, or vitamin a status biomarkers. J Nutr. 2015;145(7):1541-8.

18. Arsenault JE, Mora-Plazas M, Forero Y, et al. Micronutrient and anthropometric status indicators are associated with physical fitness in Colombian schoolchildren. Br J Nutr. 2011;105(12):1832-42.

19. García G, Trejos J, Restrepo B, Landázuri P. Homocysteine, folate and vitamin B12 in Colombian patients with coronary disease. Arq Bras Cardiol. 2007;89(2):71-6, 79-85.

20. Caicedo O, Villamor E, Forero Y, Ziade $\mathrm{J}$, et al. Relation between vitamin B12 and folate status, and hemoglobin concentration and parasitemia during acute malaria infections in Colombia. Acta Trop. 2010;114(1):17-21. doi: 10.1016/ j.actatropica.2009.11.005

21. Arsenault JE, Mora-Plazas M, Forero $\mathrm{Y}$, et al. Hemoglobin concentration is inversely associated with erythrocyte folate concentrations in Colombian school-age children, especially among children with low vitamin B12 status. Eur J Clin Nutr. 2009;63(7):842-9. doi: 10.1038/ ejcn.2008.50

22. Tavares EF, Vieira-Filho JP, Andriolo A, et al. Serum total homocysteine levels and the prevalence of folic acid deficiency and C677T mutation at the MTHFR gene in an indigenous population of Amazonia: The relationship of homocysteine with other cardiovascular risk factors. Ethn Dis. 2004;14(1):49-56.

23. Galukande M, Jombwe J, Fualal J, Baingana $\mathrm{R}$, Gakwaya A. Reference values for serum levels of folic acid and vitamin B12 in a young adult Ugandan population. Afr Health Sci. 2011;11(2):240-3.

24. Holguín-Hernández E, Orozco-Díaz JG. [Administration of folic acid and other micronutrients to pregnant women in Colombia]. Rev Panam Salud Pública. 2013;34(2):99-106.

25. Ray JG, Cole DE, Boss SC. An Ontariowide study of vitamin B12, serum folate, and red cell folate levels in relation to plasma homocysteine: is a preventable public health issue on the rise? Clin Biochem. 2000;33(5):337-43.

\section{Notas}

* Este artículo es producto del trabajo de tesis de pregrado de la Carrera de Bacteriología, de la autora principal, la bacterióloga Lyda Salamanca. 